FIG. 2 Electron transfer from putidaredoxin to cytochrome P-450 by covalent switching (adapted from ref. 1). This second type of electron transfer involves intermediate aromatic amino-acid residues (Trp, Phe or Tyr), which can allow electron transfer between sulphur atoms too far apart to interact directly. The binding of reduced putidaredoxin ( $\mathrm{Pd}$ ) to ferric P-450 is suggested ${ }^{1}$ to be accompanied by a conformational change within the putidaredoxin that moves its Cterminal tryptophan residue close enough to Cys 39 (attached to the $\mathrm{Fe}_{2} \mathrm{~S}_{2}$ cluster) to permit interaction. An ion-pair is produced that has the correct configuration to allow covalent switching with the haem-Fe-Cys ${ }^{357}$ sulphur of P-450, breaking the haem-Cys ${ }^{357}$ bond and resulting in a briefly tetracoordinate haem iron. The second stage of covalent switching transfers $\mathrm{Cys}^{357}$ from tryptophan back to haem to reform the Cys $^{357}$-haem bond, and results in overall electron transfer from putidaredoxin to the haem ring. Oxygen then binds to the haem. A second electron is delivered to the haem from reduced putidaredoxin, presumably by the same covalent switching mechanism involving tryptophan. Cleavage of the peroxo-bond may occur simultaneously, generating the substrate-hydroxylating haem-oxo-iron complex. The covalent switching breaks the $\mathrm{Cys}^{357}$-haem bond (the Cys ${ }^{357}$ is temporarily attached to $\operatorname{Trp}^{106}$ of putidaredoxin). This may be advantageous in preventing the oxo-haem from oxidizing this sulphur ligand - in the terms of Baldwin et al. ${ }^{1}$, the switch is insulational (switch on: electrons flow to haem; switch off: haem is insulated). Not until the oxo-iron has hydroxylated the substrate is the haem-Cys ${ }^{357}$ bond restored.

monas putida to illustrate the principles that may be involved. It turns out that the protein components of the enzyme are not just obstacles, but that they help to control the specificity and rate of electron transfer ${ }^{4}$ by mechanisms involving sulphur ligands, disulphide bridges and aromatic amino-acid residues ${ }^{1,4}$. In $P$. putida the cytochrome $\mathrm{P}-450$ receives electrons from putidaredoxin (a ferredoxin with an $\mathrm{Fe}_{2} \mathrm{~S}_{2}$ cluster; see Fig. 1), which in turn is reduced at the expense of NADH by a flavoprotein enzyme, the FAD-containing putidaredoxin reductase. The reduced $\mathrm{P}-450$ uses bound oxygen to hydroxylate camphor, a first step in the use of this substrate for energy production. Baldwin et al. claim that the mechanism they propose can account for the entire electron transport from NADH through to the cytochrome. The intermediate aromatic amino-acid residues crucial to the scheme of covalent switching (Fig. 2) are conserved among the many cytochromes whose sequence is known; most eukaryotic cytochromes $\mathrm{P}-450$ have specially evolved their own intramolecular tryptophan electron-transfer mediator. The covalent switch involves the redistribution of electron pairs through a framework of momentarily fixed nuclei, so is linked to the nuclear geometry of the molecular system. Baldwin et al.
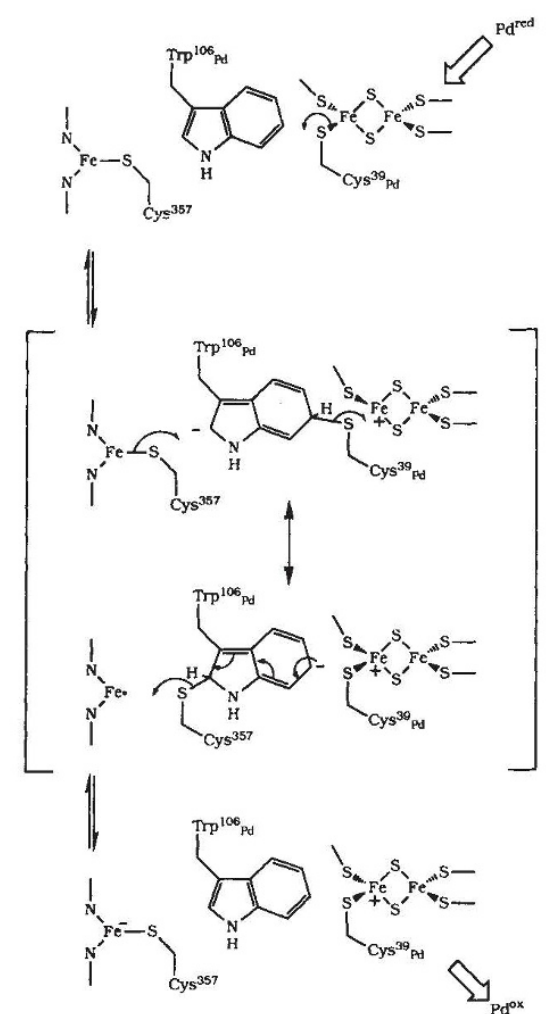

\section{Metabolic tuning}

LAST week Daedalus was contemplating reaction cycles, such as the Krebs cycle which oxidizes glucose in the body. He decided that the repetitive molecular expansion and contraction produced by such a cycle must generate a weak local sound at each site of the reaction. A strong external note at just that frequency could pull all the reaction sites in a sample of tissue into synchrony. Thereafter, the tissue would continue to sing coherently at its Krebs frequency, staying in synchrony by self-reinforcement.

Daedalus is now using this principle to control the rates of cyclic reactions. An external sonic frequency entrains a biochemical cycle by locking the molecular expansion part of the reaction into the low-pressure phase of the frequency, while the molecular contraction part is concentrated in the high-pressure phase. If the frequency is then gradually increased, the cycle will speed up to follow it; it might even reach double speed before falling out of lock with the driving frequency. Similarly, lowering the driving frequency must slow the reaction.

At a stroke, biochemists and physiologists will acquire wonderful new powers. A simple sonic driver, tuned to the crucial cyclic biochemical pathways, could double the output of industrial fermenters and brewery vats. A second driver could slow down unwanted side-reactions or sabotage interfering organisms. Crops and vegetables could be given a cunning acoustic boost - those gardeners who sing to their plants may already be operating in just this way. Medicine, too, will be transformed. Victims of exhaustion will be sung back to life by speeding their Krebs oxidation of injected glucose; parasites and infectious bacteria will be killed or weakened by slowing their metabolic rate.

Even better, this powerful treatment can be applied locally. Slimmers will welcome the DREADCO acoustic vibromassager, whose piezoelectric contact pad will magically melt their unwanted fatty tissue by speeding up its fatty-acid oxidation cycle. And the DREADCO biocyclic hat will give wonderful selective control of the brain. With its aid, poets will speed the metabolism of their creative right hemisphere, while artists selectively galvanize their visual cortex and acrobats 'tone up' their sense of balance and spatial awareness. Manics and depressives will gladly control their aberrant dopamine and noradrenaline brain chemistry. And we will all welcome the ability to slow the whole cortex into perfect, dreamless sleep: from which a smooth rise in frequency can awaken us refreshed in the morning.

David Jones 